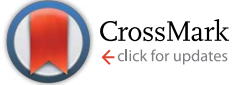

Cite this: RSC Adv., 2016, 6, 97048

\title{
A superhydrophobic hyper-cross-linked polymer synthesized at room temperature used as an efficient adsorbent for volatile organic compounds $\dagger$
}

\author{
Junhui Wang,,$^{\mathrm{ac}}$ Wan-Qiu Wang,,$^{\mathrm{b}}$ Zhengping Hao, ${ }^{a}$ Gang Wang, ${ }^{\text {ac }}$ Yang Li, ${ }^{\text {ac }}$ \\ Jian-Gang Chen, ${ }^{\mathrm{b}}$ Miaomiao $\mathrm{Li}^{\mathrm{a}}{ }^{\mathrm{j}}$ Jie Cheng ${ }^{\star \mathrm{a}}$ and Zhao-Tie Liu ${ }^{\star \mathrm{b}}$
}

Cost-effective adsorbents for volatile organic compounds elimination were synthesized at room temperature. New kinds of external cross-linkers were developed and used in the one-step FriedelCrafts reaction. The synthesized polymers exhibit a high surface area (BET surface area up to $1345 \mathrm{~m}^{2}$ $\mathrm{g}^{-1}$ ), large pore volume, superhydrophobic nature and excellent adsorption capacity for benzene, which is one of the highest to date among the reported adsorbents. It also possesses super preferential selectivity towards benzene in a high humid gas stream. Therefore, this kind of material is a promising

adsorbent for air purification and environmental protection.

Received 22nd July 2016

Accepted 23rd September 2016

DOI: $10.1039 / c 6 r a 18687 d$

www.rsc.org/advances

\section{Introduction}

Volatile organic compounds (VOCs) emitted mostly from anthropical activities pose great threat to all life forms and the environment we live in, especially in recent years. ${ }^{1,2}$ Among all the VOCs control techniques, adsorption has been recognized to be an efficient and facile recovery method to solve such severe situations. A large number of nanoporous materials have been developed as the adsorbents, e.g. carbons, silicas, resins and organic-inorganic hybrids. ${ }^{2-15}$ Among all the conventional adsorbents, activated carbon is one of the most widely used and efficient materials due to its large surface area, abundant porosity and low price. ${ }^{16}$ However, its non-selectivity towards VOCs and water vapor can greatly reduce its adsorption capacity for organic contaminants in an effluent stream where water vapor exists at a high concentration. Some commercial porous resins have also been used as adsorbents for VOCs in air and water, while low adsorption capacities and complex synthesis processes limit their development in large-scale applications. Therefore, it is still urgent to seek for a novel and efficient

${ }^{a}$ Department of Environmental Nano-materials, Research Center for Eco-Environmental Sciences, Chinese Academy of Sciences, Beijing, 100085, China. E-mail: jiecheng@rcees.ac.cn

${ }^{b}$ Key Laboratory of Applied Surface and Colloid Chemistry, School of Chemisty \& Chemical Engineering, Shananxi Normal University, Xi'an 710119, China. E-mail: ztliu@snnu.edu.cn

'University of Chinese Academy of Sciences, Beijing, 100049, China

$\dagger$ Electronic supplementary information (ESI) available. See DOI: $10.1039 / \mathrm{c} 6 \mathrm{ra} 18687 \mathrm{~d}$

$\ddagger$ These authors contributed equally to this work. material with hydrophobic nature, high adsorption capacity and facile synthesis process.

Recently, a versatile route for preparing microporous polymers using a simple one-step Friedel-Crafts reaction has been reported. ${ }^{17}$ In this approach, an external cross-linker, formaldehyde dimethyl acetal (FDA), was used and various aromatic monomers could be directly crosslinked to form highly porous networks. We subsequently observed that this "knitting" approach can produce networks with hydrophobic nature and BET surface areas of up to $1394 \mathrm{~m}^{2} \mathrm{~g}^{-1}$ when benzyl chloride was used as a monomer. ${ }^{18}$ However, these polymers mainly possessed pores narrower than $1 \mathrm{~nm}$, which are more suitable for the adsorption of $\mathrm{H}_{2}$ and $\mathrm{CO}_{2}$ instead of VOCs that have relatively larger molecular dimensions.

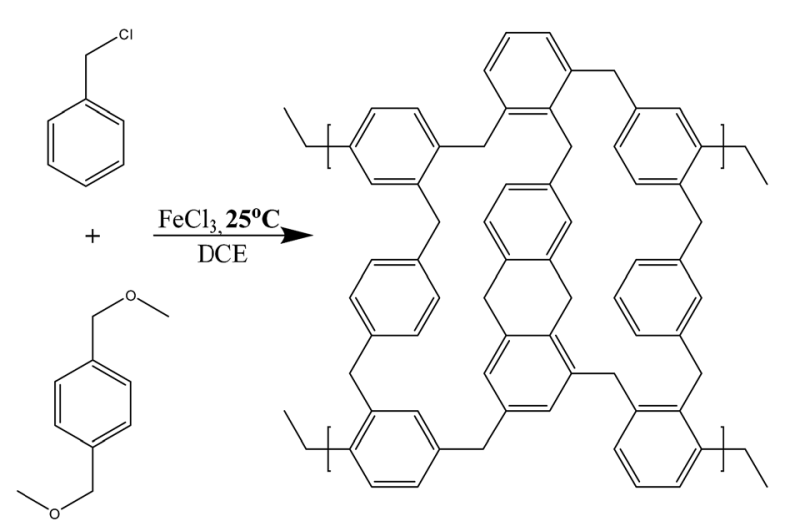

Scheme 1 Friedel-Crafts "Knitting" polymerization of benzyl chloride monomer using 1,4-bis(methoxymethyl)benzene. 
Therefore, in the present paper, we used a relatively longer linker, 1,4-bis(methoxymethyl)benzene, and benzyl chloride as the monomer to synthesize hyper-crosslinked polymers (HCPs) (Scheme 1) via the Friedel-Crafts reaction. Porous polymers with very high surface areas can be synthesized at a room temperature. To the best of our knowledge, this is the first report on the synthesis of HCPs at room temperature, which makes the procedure easier and much environmentally friendly. Moreover, this type of HCPs displayed extraordinary adsorption capacities towards organic molecules like benzene and have superhydrophobic nature.

\section{Experimental section}

\section{Synthesis of ClBE- $x$}

In a typical synthesis, 1,4-bis(methoxymethyl)benzene was used as an external cross-linker to react with the aromatic monomer, benzyl chloride. Typically, $0.63 \mathrm{~g}$ of benzyl chloride $(5 \mathrm{mmol})$ and various amounts of 1,4-bis(methoxymethyl)benzene $(y$ $\mathrm{mmol}$ ) were added to 1,2-dichloroethane (DCE, $20 \mathrm{~mL}$ ), followed by iron(II) chloride (10 $\mathrm{mmol})$. After that, the mixture was stirred at a room temperature for $18 \mathrm{~h}$, after which the solid product was removed by filtration and washed with methanol and ether until the filtrate was clear. The product was then further purified by Soxhlet extraction in methanol for $12 \mathrm{~h}$ and dried in vacuum at $60^{\circ} \mathrm{C}$ for $24 \mathrm{~h}$. All the samples were obtained as light or dark brown powders and denoted as ClBE- $x$, where $x$ refers to the molar ratio of the cross-linker to the monomer.

\section{Characterization and measurements}

The porous properties of each sample were investigated by nitrogen adsorption tests at liquid nitrogen temperature (77 K) on a Micromeritics ASAP2020 analyzer. The Fourier transform infrared (FT-IR) spectra were measured on a Bruker Tensor 27 spectrometer in the range of $600-4000 \mathrm{~cm}^{-1}$. The solid-state NMR spectra were measured on a Bruker BioSpin GmbH spectrometer operating at $151 \mathrm{MHz}$ for ${ }^{13} \mathrm{C}$. The morphologies of samples were inspected using field emission scanning electron microscopy (FE-SEM, SU-8020, Japan). Thermogravimetric analysis (TGA) of the adsorbents was performed on a TG/DTA analyzer (Setaram, Labsys) at a heating rate of $10{ }^{\circ} \mathrm{C} \mathrm{min}{ }^{-1}$ from 30 to $800{ }^{\circ} \mathrm{C}$ under a nitrogen flow of $60 \mathrm{~mL} \mathrm{~min}{ }^{-1}$. The water sessile drop contact angle (CA) was used to examine the hydrophobicity of the synthesized polymers using the OCA $15 \mathrm{EC}$ video based contact angle measuring device (Dataphysics, Germany) at room temperature. Before measurement, the powdered sample was pressed using a tableting machine to guarantee a plane surface of the sample.

\section{Adsorption studies}

The static adsorption characteristics of benzene and water on the adsorbents in this study were investigated using an intelligent gravimetric analyzer (Model IGA-002, HidenIsochema Instrument). The apparatus had an ultra-high vacuum system allowing the isotherms to be determined by setting pressure steps. Before measurement, the adsorbent sample (60 $\pm 1 \mathrm{mg}$ ) was outgassed to a constant weight at $373 \mathrm{~K}$ under a pressure of $<10^{-6} \mathrm{~Pa}$.

Dynamic adsorption measurements were carried out on a fixed-bed column and the corresponding experimental set-up and method are described in our previous work. ${ }^{19}$ The samples were degassed at $80{ }^{\circ} \mathrm{C}$ overnight to remove any possible impurities before each adsorption test. Before starting each

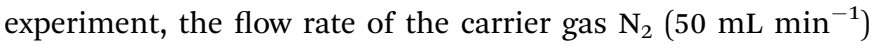
was adjusted so that the outlet concentration of benzene vapor reached $550 \mathrm{ppm}$ and remained stable. In order to investigate the effect of water vapor on the adsorption behavior, $550 \mathrm{ppm}$ of benzene under a relative humidity $(\mathrm{RH})$ of $80 \%$ was passed through the adsorption bed. The benzene concentration was recorded on a gas chromatograph (GC) equipped with a flame ionization detector and the breakthrough curves were collected. The adsorbed amounts were calculated using the following equation: ${ }^{20}$

$$
Q=\frac{F_{\mathrm{A}} t_{\mathrm{q}}}{W}
$$

The time $t_{\mathrm{q}}$ was calculated according to eqn (2):

$$
t_{\mathrm{q}}=\int\left(1-\frac{C_{0}}{C_{\mathrm{i}}}\right) \mathrm{d} t-t_{\mathrm{D}}
$$

where $Q$ is the adsorbed amount $\left(\mathrm{mmol} \mathrm{g}^{-1}\right) ; F_{\mathrm{A}}$ is the molar flow of the gas $\left(\mathrm{mol} \mathrm{min}^{-1}\right)$; $W$ is the net weight of the adsorbent (g); $C_{\mathrm{i}}$ and $C_{0}$ represent the benzene concentration at the inlet and outlet $\left(\mathrm{g} \mathrm{mL}^{-1}\right)$, respectively, and $t_{\mathrm{D}}$ is the dead time of the system (min).

\section{Results and discussion}

\section{Characterization of adsorbents}

In the presence of the catalyst, the cleavage of $\mathrm{C}-\mathrm{O}$ bond generates a methoxy group, which acts as a leaving group. Afterwards, the intermediate can react with both the monomer and itself leading to the structure displayed in Scheme 1. The chemical structures of the polymers synthesized were initially confirmed by Fourier transform infrared (FTIR) spectroscopy and ${ }^{13} \mathrm{C}$ cross-polarization magic-angle spinning (CP/MAS) NMR spectroscopy. In the FTIR spectra of all the samples (Fig. S1 $\dagger$ ), the bands at 1601, 1500 and $1436 \mathrm{~cm}^{-1}$ were attributed to the $-\mathrm{C}=\mathrm{C}-$ vibration of the aromatic ring. The vibration peaks at $2913 \mathrm{~cm}^{-1}$ and $3010 \mathrm{~cm}^{-1}$ are identified as the $\mathrm{CH}$ stretching vibration of $-\mathrm{CH}_{2}$ - and the phenylene group. ${ }^{21}$ The bands at 1089 and $1265 \mathrm{~cm}^{-1}$ were assigned to $\mathrm{C}-\mathrm{O}$ stretching vibration and $\mathrm{CH}_{2} \mathrm{Cl}$ group. ${ }^{22,23}$ The ${ }^{13} \mathrm{C} \mathrm{CP} / \mathrm{MAS}$ NMR of ClBE1.5 (Fig. S2 $\dagger$ ) shows resonance peaks near 136 and 129 ppm due to the substituted aromatic carbons and non-substituted aromatic carbons, and the resonance peak near 36 ppm was assigned to the carbon in the methylene linker formed after the Friedel-Crafts reaction. ${ }^{\mathbf{1 7}}$ The characterization data indicate that the expected polymers were successfully synthesized.

The morphology of the samples was investigated by SEM as shown in Fig. 1. The images indicate the formation of amorphous particles with a size of approximately $50-100 \mathrm{~nm}$ in 


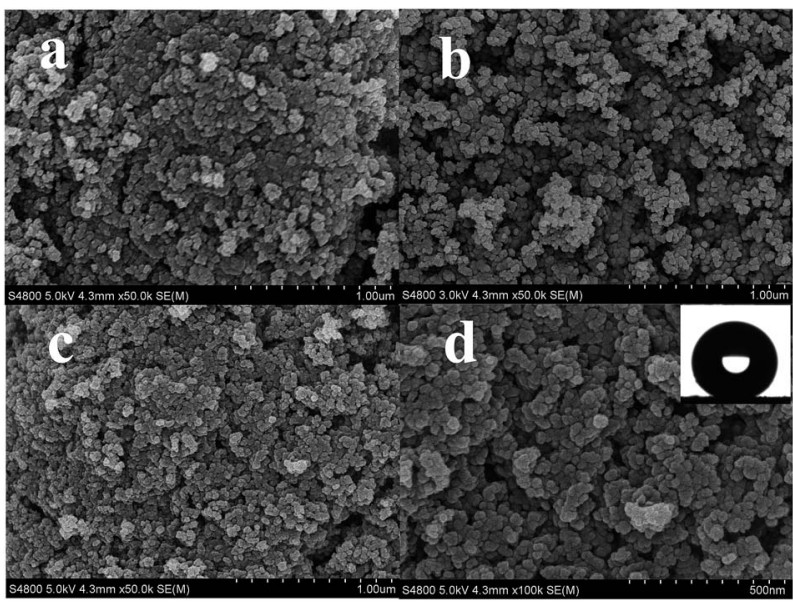

Fig. 1 SEM images of CIBE-0.5 (a), CIBE-1 (b), CIBE-1.5 (c) and (d); the inset is a photograph of a water droplet on a tablet of CIBE-1.5.

diameter, which is similar to other types of hyper-crosslinked polymers. ${ }^{17,24}$ Further analysis of the polymers by thermogravimetric analysis (TGA) showed that the sample has great thermal stability with a thermal decomposition temperature over $550{ }^{\circ} \mathrm{C}$ (Fig. S3†) due to the strong covalent bonding in the network. The slight weight loss of approximately $5 \%$ before $550{ }^{\circ} \mathrm{C}$ may be attributed to the desorption of the adsorbed monomers or molecules of solvent confined inside the pore channels of the synthesized HCP. Moreover, all the polymers were insoluble in common organic solvents such as alkanes, alcohols, acetone and DMF, which suggests their high chemical stability.

The porous properties of the samples were analysed by nitrogen adsorption and desorption at liquid nitrogen temperature $(77 \mathrm{~K})$. Table 1 summarizes the characterization data such as surface area and the pore volume. The highest BET surface area $\left(1345 \mathrm{~m}^{2} \mathrm{~g}^{-1}\right)$ was obtained when the molar ratio of crosslinker to monomer was 1.5 , because of the high crosslinking extent. A higher cross-linker content can lead to a higher surface area and total pore volume, but an appropriate ratio is necessary to obtain a high micropore volume. The apparent BET value was relatively high when compared to the other previously reported HCPs and was much higher than other organic polymers. ${ }^{17,24-28}$ As shown in Fig. 2a, the adsorption isotherms obtained for all the samples indicate a steep nitrogen gas uptake $\left(>100 \mathrm{~cm}^{3} \mathrm{~g}^{-1}\right)$ at low relative pressure $\left(P / P_{0}<0.001\right)$, thus reflecting an abundant microporous structure. ${ }^{29}$ The existence of the typical hysteresis loops indicates mesoporous structures and the sharp rise in the adsorption extent in the medium and high pressure region $\left(P / P_{0}=0.8-1.0\right)$ suggests the presence of macropores in

Table 1 The textural properties of the samples

\begin{tabular}{lllll}
\hline Material & $S_{\text {BET }}\left(\mathrm{m}^{2} \mathrm{~g}^{-1}\right)$ & $V_{\text {tot }}\left(\mathrm{cm}^{3} \mathrm{~g}^{-1}\right)$ & $V_{\text {mic }}\left(\mathrm{cm}^{3} \mathrm{~g}^{-1}\right)$ & $S_{\text {mic }}\left(\mathrm{cm}^{2} \mathrm{~g}^{-1}\right)$ \\
\hline ClBE-0.5 & 894 & 0.88 & 0.09 & 218 \\
ClBE-1.0 & 1247 & 1.92 & 0.06 & 167 \\
ClBE-1.5 & 1345 & 1.75 & 0.09 & 225 \\
ClBI & 1017 & 0.83 & 0.14 & 318
\end{tabular}
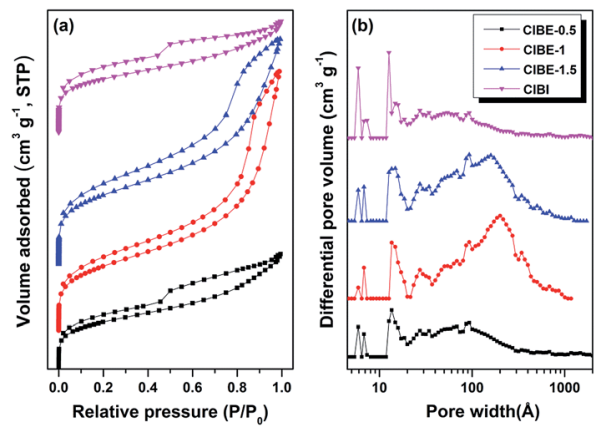

Fig. 2 The nitrogen adsorption/desorption isotherms obtained at $77 \mathrm{~K}$ (a) and the pore size distribution calculated using DFT methods (b) of the samples.

ClBE-1 and ClBE-1.5, which can also be observed in Fig. 2 b. ${ }^{29}$ The pore size distribution also confirms the presence of such a heterogeneous porous structure. Furthermore, 4,4'-bis(methoxymethyl)biphenyl was also used as an external cross-linker to synthesize the hyper-crosslinked polymers, named ClBI. Its porous properties are displayed in Fig. 2 and are listed in Table 1. It can be observed that this sample also possesses a high surface area and relatively more micropores.

\section{Adsorption isotherms}

For comparison, the adsorption and desorption isotherms of ClBE-1.5, which has the highest BET surface area and microporous volume, and commercial resin XAD-4 (BET surface area $760 \mathrm{~m}^{2} \mathrm{~g}^{-1}$, see Fig. S5†) for benzene as a function of relative pressure at $25{ }^{\circ} \mathrm{C}$ are shown in Fig. 3. Apparently, the adsorbed amounts of benzene for ClBE-1.5 are all higher than those observed for XAD-4 over the entire pressure range studied. In addition, ClBE-1.5 shows a higher adsorption capacity than the other commonly used adsorbents, such as MOFs, resins, zeolites and activated carbon together with their derivatives (shown in Table 2). For example, ClBE-1.5 adsorbs $17.85 \mathrm{mmol}$ $\mathrm{g}^{-1}$ of benzene at $25^{\circ} \mathrm{C}$ and $0.8 P / P_{0}$, which was twice the value obtained for SBA-15, activated carbon and their derivatives (ACFC, ACC-963) and 3-5 times larger than that of NDA-201 resin and HZSM-5 zeolite (Table 2). To the best of our

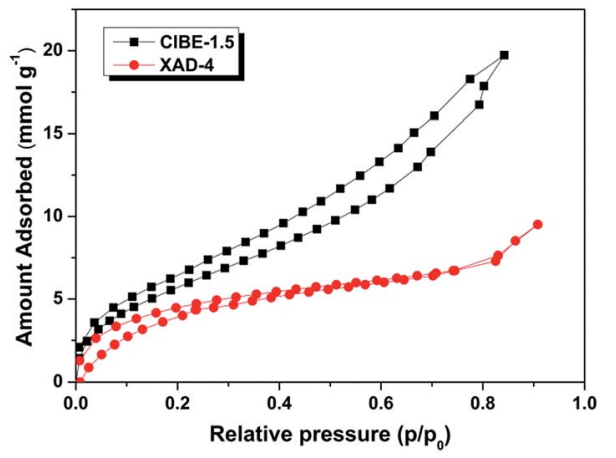

Fig. 3 The adsorption and desorption isotherms of benzene on CIBE1.5 and XAD- 4 at $25^{\circ} \mathrm{C}$. 
Table 2 The adsorption capacities for benzene obtained for CIBE-1.5 and selected adsorbents reported in the literature ${ }^{a}$

\begin{tabular}{|c|c|c|c|c|c|c|}
\hline \multirow[b]{2}{*}{ Adsorbent } & \multirow[b]{2}{*}{$S_{\mathrm{BET}}\left(\mathrm{m}^{2} \mathrm{~g}^{-1}\right)$} & \multirow[b]{2}{*}{$V_{\text {tot }}\left(\mathrm{cm}^{3} \mathrm{~g}^{-1}\right)$} & \multirow[b]{2}{*}{$Q_{\mathrm{a}}\left(\mathrm{mmol} \mathrm{g}^{-1}\right)$} & \multicolumn{2}{|c|}{ Conditions } & \multirow[b]{2}{*}{ Ref. } \\
\hline & & & & $C_{\mathrm{e}}\left(P / P_{0}\right)$ & $T\left({ }^{\circ} \mathrm{C}\right)$ & \\
\hline ClBE-1.5 & 1345 & 1.75 & 17.85 & 0.8 & 25 & This work \\
\hline MIL-101 & 3980 & 1.85 & 16.55 & 0.8 & 25 & 13 \\
\hline Activated carbon & 1600 & 2.00 & 8.00 & 0.8 & 30 & 14 \\
\hline ACFC & 1604 & NA & 8.13 & 0.8 & 20 & 31 \\
\hline ACC-963 & 1705 & 0.67 & 7.80 & 0.8 & 0 & 32 \\
\hline SBA-15 & 805 & 0.97 & 7.00 & 0.8 & 30 & 14 \\
\hline HZSM-5 & 550 & 0.20 & 1.90 & 0.8 & 30 & 33 \\
\hline NDA-201 resin & 855.6 & 0.52 & 5.20 & 0.8 & 30 & 25 \\
\hline HCP-0.5 & 1394 & 1.55 & 14.20 & 0.8 & 25 & 18 \\
\hline HCP-1.3 & 1231 & 1.99 & 16.00 & 0.8 & 25 & 30 \\
\hline
\end{tabular}

${ }^{a} S_{\mathrm{BET}}$ is the specific surface area calculated using the BET method; $V_{\mathrm{tot}}$ is the total pore volume; $Q_{\mathrm{a}}$ is the reported data for the amount of benzene adsorbed under specified conditions; $C_{\mathrm{e}}$ is the relative pressure $\left(P / P_{0}\right)$ at which the reported $Q_{\mathrm{a}}$ values were obtained; $T$ is the temperature at which the reported $Q_{\mathrm{a}}$ values were obtained.

knowledge, the maximum adsorption capacity of benzene reported in the literature ${ }^{13}$ is $16.55 \mathrm{mmol} \mathrm{g}^{-1}$ on MIL-101 (one kind of metal-organic frameworks) with a large surface area of $3980 \mathrm{~m}^{2} \mathrm{~g}^{-1}$. Such high adsorption capacity for benzene by ClBE-1.5 can be attributed to the cooperation of both its large pore volume and the structural similarity between the adsorbate and adsorbent (comprised of skeletal aromatic rings). ${ }^{30}$

For the purpose of obtaining direct information on the surface hydrophobicity of adsorbents discussed, water contact angle measurements and water vapor adsorption experiments were conducted. It was observed that ClBE-1.5 exhibits surface superhydrophobicity with a water CA of $150.6^{\circ}$ (Fig. 1d and S4 $\dagger$ ) (the surface with water contact angle larger than $150^{\circ}$ is called superhydrophobic). ${ }^{34}$ The isotherms for water adsorption on ClBE-1.5 and XAD-4 at $25{ }^{\circ} \mathrm{C}$ are shown in Fig. 4. It is well recognized that water molecules adsorb primarily on the hydrophilic sites at low pressure, followed by the formation of clusters or a continuous adsorption film and pore filling occurs at high pressure. The adsorption isotherm is a function of the concentration and distribution of primary adsorption centers, the pore structure and the vapor pressure. ${ }^{35}$ The uptake of water vapor at low pressure $\left(P / P_{0}\right.$ is below 0.3$)$ relies on the

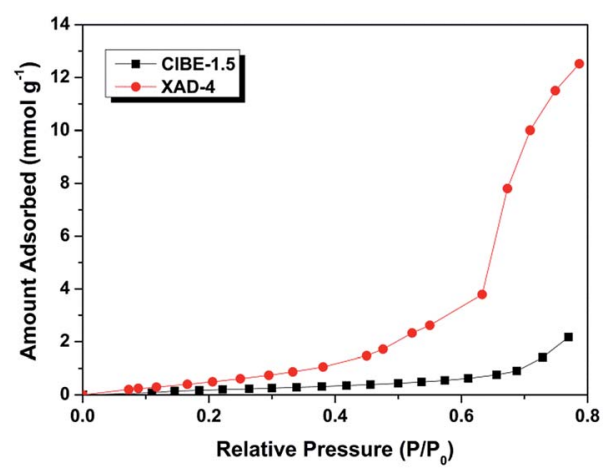

Fig. 4 The adsorption isotherms for water vapor on CIBE-1.5 and XAD-4 obtained at $25^{\circ} \mathrm{C}$. concentration of hydrophilic sites and at high pressure is related to the pore structure of the adsorbents. ${ }^{3}$ In other words, at low pressure, the more hydrophobic the adsorbent is, the lower uptake of water vapor it has. It is obvious that ClBE-1.5 retains a much lower uptake of water vapor no matter at low or high pressure as shown in Fig. 4. These results directly indicate the fact that ClBE-1.5 possesses a superhydrophobic surface.

\section{Adsorbate-adsorbent interaction}

In order to quantitatively evaluate the affinity between the adsorbent and adsorbate, the Henry constants were calculated, which can be obtained from the virial equation: ${ }^{36}$

$$
\ln (n / p)=A_{0}+A_{1} n+A_{2} n^{2}+\ldots
$$

where $n$ is the amount adsorbed $\left(\mathrm{mol} \mathrm{g}^{-1}\right)$ at pressure $p(\mathrm{~Pa})$ and the first virial coefficient $A_{0}$ is related to the Henry constant, $K_{\mathrm{H}}$, by the equation $K_{\mathrm{H}}=\exp \left(A_{0}\right)$. $K_{\mathrm{H}}$ depends on the interaction between the adsorbent surface and the adsorbed gas molecule. The higher terms $\left(A_{2}\right.$, etc. $)$ can be ignored and a linear graph of $\ln (n / p)$ versus $n$ is obtained at a low surface coverage.

Fig. S6 $\uparrow$ shows the virial graphs for benzene on ClBE-1.5 and $\mathrm{XAD}-4$ at $25{ }^{\circ} \mathrm{C}$. The Henry constants calculated from parameter

Table 3 The Henry constants for benzene and water on CIBE-1.5, $X A D-4$ and $A C$

\begin{tabular}{llrl}
\hline & \multicolumn{2}{l}{$\begin{array}{l}\text { Henry constant } K_{\mathrm{H}} \\
\left(\times 10^{-7} \mathrm{~mol} \mathrm{~g}^{-1} \mathrm{~Pa}^{-1}\right)\end{array}$} & \\
\cline { 2 - 3 } & Benzene & Water & \multicolumn{1}{l}{$\begin{array}{l}\text { Selectivity } \\
\text { benzene/water }\end{array}$} \\
\hline Adsorbent & 283.75 & 2.38 & 119.20 \\
ClBE-1.5 & 37.64 & 8.56 & 4.40 \\
$\mathrm{XAD}^{a}$ & 442.79 & 12.04 & 36.78
\end{tabular}

${ }^{a}$ The data for AC are cited from ref. 12 . 
$A_{0}$ of benzene and water on ClBE-1.5 and XAD- 4 at $25{ }^{\circ} \mathrm{C}$ are listed in Table 3. As for benzene, the Henry constants increase in the order of $\mathrm{AC}>\mathrm{ClBE}-1.5>\mathrm{XAD}-4$, which can be explained by the strong dispersion interactions in the micropores. In the literature, $\mathrm{Hu}$ et al. have pointed out that benzene is more likely to adsorb on the adsorbents, which are full of benzene rings. That is why ClBE-1.5 exhibits a high affinity towards benzene, even though it has a small amount of micropores. ${ }^{19}$ While for water, the Henry constant of water on ClBE-1.5 was smaller than those on both XAD-4 and AC. This finding supports the abovementioned conclusion that ClBE-1.5 holds a hydrophobic surface. Meanwhile, the ratio of the Henry constant between benzene and water on ClBE-1.5 was as high as 119, which indicates the high selectivity between VOCs gases and water vapor of this adsorbent.

\section{Isosteric heat of adsorption}

The change in the isosteric enthalpy with the surface loading can provide useful information on the molecular scale interactions between the adsorbent and adsorbate molecules, and also can be used as a measure of the energetic heterogeneity of solid surface. If the surface is homogeneous, the heat of adsorption is constant at all loadings. However, in the case of an energetically heterogeneous surface, the isosteric enthalpy of adsorption varies with the surface loading. The heat of adsorption decreases with the increasing number of moles adsorbed, if there is no interaction between the adsorbed molecules or the interaction is negligible compared to that between the adsorbent and adsorbate. On the other hand, if the adsorbate-adsorbate interaction is high or comparable with the adsorbate-adsorbent interaction, the heat of adsorption increases at high coverage. ${ }^{37}$

The isosteric enthalpies $\left(\Delta H_{\mathrm{i}}\right)$ of adsorption can be calculated at a constant surface coverage using the equation shown below, if a set of adsorption isotherms was obtained at several temperatures $\left(25^{\circ} \mathrm{C}, 35^{\circ} \mathrm{C}\right.$ and $\left.45^{\circ} \mathrm{C}\right):{ }^{36}$

$$
\ln (p)=\Delta H_{\mathrm{i}} / R T-\Delta S_{\mathrm{i}} / R
$$

where $p$ is the pressure, $R$ is the gas constant and $T$ is the temperature. In Fig. 5, the isosteric enthalpy of adsorption for the two adsorbents calculated using Freundlich isotherm model

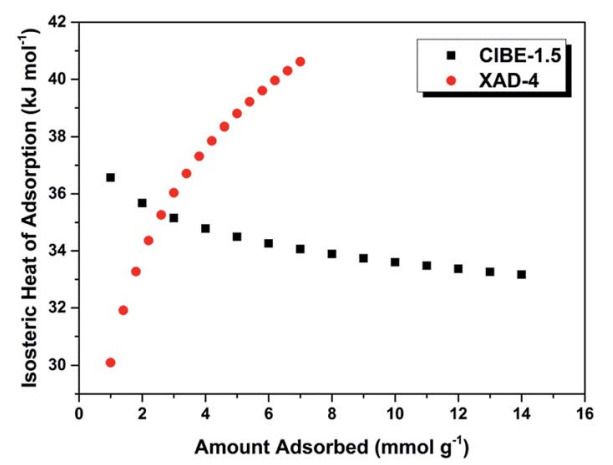

Fig. 5 The isosteric enthalpies for benzene adsorption on CIBE-1.5 and XAD-4. was plotted as a function of the amount adsorbed. As shown in Fig. 5, the isosteric heat of adsorption varies with the coverage, which means the two adsorbents both have energetically heterogeneous surfaces. Moreover, it can be observed that the isosteric heat at lower coverage for ClBE-1.5 was higher than that found for XAD-4, which again proves the higher affinity between ClBE-1.5 and benzene. As for the change in the isosteric enthalpy of adsorption, the two adsorbents show quite different trends. Because the adsorbate-adsorbate lateral interactions increase as the coverage increases and for XAD-4, the benzene-benzene interaction is not negligible when compared with the low benzene-XAD-4 affinity.

\section{Breakthrough curve}

In order to examine the effect of water vapor in the stream on the adsorption performance of VOCs, the dynamic experiments of benzene adsorption onto the representative samples of ClBE1.5 and XAD- 4 were conducted. A breakthrough measurement is a direct method designed to determine the dynamic performance of VOC adsorption at low concentration. ${ }^{38}$ Fig. 6 shows the adsorption breakthrough curves of benzene on ClBE-1.5 and XAD-4 under dry conditions $(\mathrm{RH}=0 \%)$ and humid conditions $(\mathrm{RH}=80 \%)$. To quantitatively elucidate the effect of relative humidity on the adsorption, the Yoon and Nelson model ( $\mathrm{Y}-\mathrm{N}$ model) ${ }^{19}$ was used to simulate the breakthrough curves according to the following equation:

$$
\frac{C_{0}}{C_{\mathrm{i}}}=\frac{1}{1+\exp \left[K^{\prime}\left(\tau_{0}-t\right)\right]}
$$
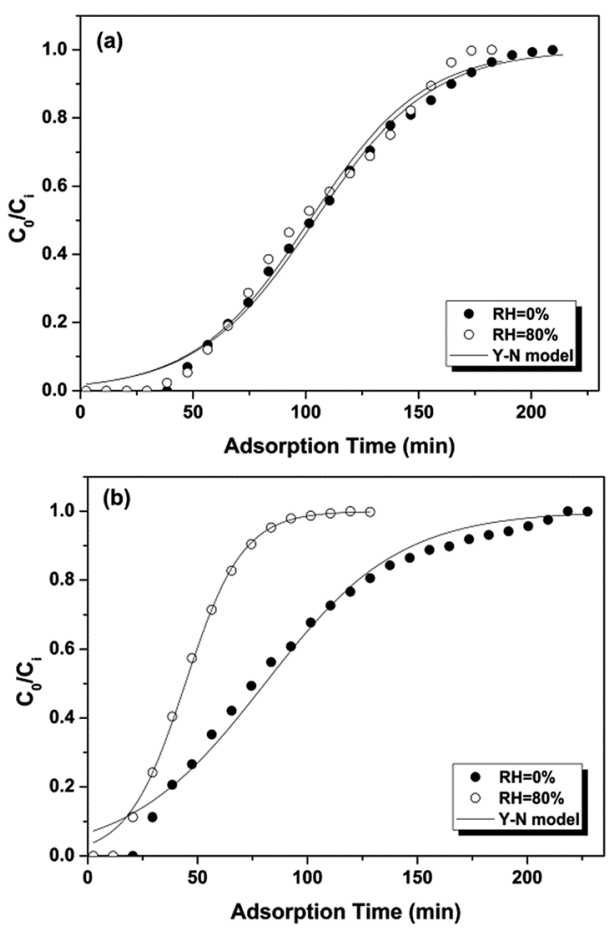

Fig. 6 The breakthrough curves obtained for benzene adsorption on CIBE-1.5 (a) and XAD-4 (b). 
Table 4 The breakthrough adsorption data for benzene compiled from the literature

\begin{tabular}{|c|c|c|c|c|c|c|c|c|}
\hline \multirow[b]{2}{*}{ Adsorbent } & \multirow{2}{*}{$\begin{array}{l}S_{\mathrm{BET}} \\
\left(\mathrm{m}^{2} \mathrm{~g}^{-1}\right)\end{array}$} & \multirow{2}{*}{$\begin{array}{l}\text { Temperature } \\
\left({ }^{\circ} \mathrm{C}\right)\end{array}$} & \multirow{2}{*}{$\begin{array}{l}\text { Concentration } \\
\text { (ppmv) }\end{array}$} & \multirow{2}{*}{$\begin{array}{l}\text { Humidity } \\
\text { RH }\end{array}$} & \multicolumn{2}{|c|}{$\begin{array}{l}Q(\mathrm{~g} / 100 \mathrm{~g} \\
\text { adsorbent) }\end{array}$} & \multirow[b]{2}{*}{$Q_{\mathrm{h}} / Q_{\mathrm{d}}{ }^{a}$} & \multirow[b]{2}{*}{ Ref. } \\
\hline & & & & & Dry & Humid & & \\
\hline ACC & NA & NA & 500 & $86 \%$ & 3.5 & 2.2 & $63 \%$ & 39 \\
\hline \multirow[t]{4}{*}{$\mathrm{AC}$} & $1100-1200$ & 24 & 400 & $60 \%$ & 30 & 19.1 & $64 \%$ & 40 \\
\hline & & & & $90 \%$ & & 8.7 & $29 \%$ & \\
\hline & & & 600 & $60 \%$ & 34.3 & 24.9 & $73 \%$ & \\
\hline & & & & $90 \%$ & & 12.1 & $35 \%$ & \\
\hline AC & 704 & 25 & 600 & $80 \%$ & 13.4 & 7.0 & $52 \%$ & 12 \\
\hline HCP-0.5 & 1394 & 25 & 530 & $80 \%$ & 13.3 & 11.4 & $86 \%$ & 18 \\
\hline MSN-100 & 578 & 20 & 800 & $80 \%$ & 6.9 & 5.6 & $81 \%$ & 41 \\
\hline CSA-2 & 727 & 25 & 700 & $18 \%$ & 12.3 & 10.4 & $85 \%$ & 6 \\
\hline ClBE-1.5 & 1345 & 25 & 550 & $80 \%$ & 14.9 & 14.5 & $97 \%$ & This work \\
\hline XAD-4 & 760 & 25 & 550 & $80 \%$ & 11.5 & 6.63 & $57 \%$ & This work \\
\hline
\end{tabular}

where $C_{\mathrm{i}}$ and $C_{0}$ are the inlet and outlet concentration of benzene in the stream through the fixed bed column $\left(\mathrm{mg} \mathrm{L}^{-1}\right), t$ is the adsorption time ( $\mathrm{min}), \tau_{0}$ is the time required for $50 \%$ adsorbate breakthrough ( $\mathrm{min}$ ) and $K^{\prime}$ is a rate constant that depends on the diffusion characteristics of the mass transfer zone $\left(\min ^{-1}\right)$.

Fig. 6 and Table S1† show that the breakthrough curves of benzene on ClBE-1.5 and XAD-4 under dry conditions and under humid conditions ( $\mathrm{RH}=80 \%$ ) were well fitted by the $\mathrm{Y}-\mathrm{N}$ model. It can be clearly observed that the presence of water vapor almost did not change the breakthrough curve of the ClBE-1.5 adsorbent when the relative humidity was increased from 0 to $80 \%$, while it is quite different for XAD-4. For the purpose of a measurable comparison, the calculated adsorption capacities and breakthrough times based on the $\mathrm{Y}-\mathrm{N}$ equation are presented in Table S1. $\dagger$ The breakthrough time is defined as the time at which the outlet concentration is $5 \%$ of the inlet concentration. From Table $\mathrm{S} 1, \dagger$ we can see under dry and humid conditions, the benzene adsorption capacities of ClBE1.5 are both higher than those found for XAD-4, which indicates the high dynamic capacity of this new type of adsorbent. Meanwhile, the presence of a high concentration of water vapor in the gaseous mixture does not induce any significant reduction in the adsorption capacity of benzene on ClBE-1.5; the adsorption efficiency $\left(Q_{\text {wet }} / Q_{\text {dry }}\right.$ ) was $97 \%$. On the contrary, the decrease in the adsorption capacity of XAD- 4 was as high as $43 \%$ when the relative humidity was increased from $0 \%$ to $80 \%$. Due to the different experimental conditions employed, it is difficult to obtain a direct comparison with the results obtained for the adsorption capacity using the different adsorbents published in the literature. Nevertheless, taking into account the breakthrough curves measured under similar experimental conditions to those employed in the present work, the adsorption capacity values under practical conditions are presented in Table 4. The data obtained for different kinds of adsorbents, such as activated carbon (ACC, AC), polymer adsorbent (HCP0.5), zeolite (MSN-100) and carbon-silica composites (CSA-2) are included. It can be observed that, the adsorption capacity for benzene on ClBE-1.5 was comparable to those obtained using other adsorbents and ClBE-1.5 shows higher hydrophobicity. Hence, it is expected that ClBE-1.5 will be a promising adsorbent for the removal of VOCs from high relatively humid streams.

\section{Conclusions}

In summary, we have synthesized superhydrophobic polymers using new kinds of cross-linker at a room temperature. These materials possess and exhibit micro/mesoporous structures, large surface areas and superhydrophobicity. In the adsorption experiments, the hyper-cross-linked polymer revealed a high adsorption capacity and preferential selectivity for benzene when compared with commercial adsorbents such as activated carbon and resin. In general, the hydrophobic nature, high adsorption capacity and high thermal stability up $550{ }^{\circ} \mathrm{C}$ imply this kind of material is a promising VOCs adsorbent and can be applied in VOCs control under humid conditions.

\section{Acknowledgements}

This work was financially supported by the National Natural Science Foundation $(21337003,21327011,21477149)$, the Strategic Priority Research Program of the Chinese Academy of Sciences (No. XDB05050200) and National key research and development program (2016YFC0204203).

\section{Notes and references}

1 A. Amari, M. Chlendi, A. Gannouni and A. Bellagi, Ind. Eng. Chem. Res., 2010, 49, 11587-11593.

2 N. Qi, W. S. Appel, M. D. LeVan and J. E. Finn, Ind. Eng. Chem. Res., 2006, 45, 2303-2314.

3 T. Karanfil and S. A. Dastgheib, Environ. Sci. Technol., 2004, 38, 5834-5841.

4 A. B. Fuertes, G. Marban and D. M. Nevskaia, Carbon, 2003, 41, 87-96. 
5 B. Dou, J. Li, Q. Hu, C. Ma, C. He, P. Li, Q. Hu, Z. Hao and S. Qiao, Microporous Mesoporous Mater., 2010, 133, 115-123.

6 B. Dou, J. Li, Y. Wang, H. Wang, C. Ma and Z. Hao, J. Hazard. Mater., 2011, 196, 194-200.

7 M. Kishima, H. Mizuhata and T. Okubo, J. Phys. Chem. B, 2006, 110, 13889-13896.

8 J. Y. Ying, C. P. Mehnert and M. S. Wong, Angew. Chem., Int. Ed., 1999, 38, 56-77.

9 C. Long, P. Liu, Y. Li, A. M. Li and Q. X. Zhang, Environ. Sci. Technol., 2011, 45, 4506-4512.

10 E. C. Peters, F. Svec and J. M. J. Frechet, Adv. Mater., 1999, 11, 1169-1181.

11 Y. Zhang, S. Wei, F. Liu, Y. Du, S. Liu, Y. Ji, T. Yokoi, T. Tatsumi and F.-S. Xiao, Nano Today, 2009, 4, 135-142.

12 J. H. Wang, G. Wang, W. Q. Wang, Z. S. Zhang, Z. T. Liu and Z. P. Hao, J. Mater. Chem. A, 2014, 2, 14028-14037.

13 K. Yang, Q. Sun, F. Xue and D. H. Lin, J. Hazard. Mater., 2011, 195, 124-131.

14 S. H. Jhung, J. H. Lee, J. W. Yoon, C. Serre, G. Ferey and J. S. Chang, Adv. Mater., 2007, 19, 121-124.

15 L. Zhang, H. C. L. Abbenhuis, Q. H. Yang, Y. M. Wang, P. C. M. M. Magusin, B. Mezari, R. A. van Santen and C. Li, Angew. Chem., Int. Ed., 2007, 46, 5003-5006.

16 A. J. Fletcher and K. M. Thomas, Langmuir, 1999, 15, 69086914.

17 B. Li, R. Gong, W. Wang, X. Huang, W. Zhang, H. Li, C. Hu and B. Tan, Macromolecules, 2011, 44, 2410-2414.

18 W.-Q. Wang, J. Wang, J.-G. Chen, X.-S. Fan, Z.-T. Liu, Z.-W. Liu, J. Jiang and Z. Hao, Chem. Eng. J., 2015, 281, 34-41.

19 Q. Hu, J. J. Li, Z. P. Hao, L. D. Li and S. Z. Qiao, Chem. Eng. J., 2009, 149, 281-288.

20 R. Serna-Guerrero and A. Sayari, Environ. Sci. Technol., 2007, 41, 4761-4766.

21 C. Li, J. Liu, X. Shi, J. Yang and Q. Yang, J. Phys. Chem. C, 2007, 111, 10948-10954.

22 A. M. Puziy, O. I. Poddubnaya, A. Martinez-Alonso, F. SuarezGarcia and J. M. D. Tascon, Carbon, 2002, 40, 1493-1505.

23 J. H. Huang, X. Y. Jin, J. L. Mao, B. Yuan, R. J. Deng and S. G. Deng, J. Hazard. Mater., 2012, 217, 406-415.
24 Y. L. Luo, B. Y. Li, W. Wang, K. B. Wu and B. Tan, Adv. Mater., 2012, 24, 5703-5707.

25 J. Y. Lee, C. D. Wood, D. Bradshaw, M. J. Rosseinsky and A. I. Cooper, Chem. Commun., 2006, 25, 2670-2672.

26 R. Dawson, L. A. Stevens, T. C. Drage, C. E. Snape, M. W. Smith, D. J. Adams and A. I. Cooper, J. Am. Chem. Soc., 2012, 134, 10741-10744.

27 J. X. Jiang, F. Su, H. Niu, C. D. Wood, N. L. Campbell, Y. Z. Khimyak and A. I. Cooper, Chem. Commun., 2008, 4, 486-488.

28 N. B. McKeown, B. S. Ghanem, K. J. Msayib, P. M. Budd, C. E. Tattershall, K. Mahmood, S. Tan, D. Book, H. W. Langmi and A. Walton, Angew. Chem., Int. Ed., 2006, 45, 1804-1807.

29 R. Pierotti and J. Rouquerol, Pure Appl. Chem., 1985, 57, 603619.

30 G. Wang, B. Dou, J. Wang, W. Wang and Z. Hao, RSC Adv., 2013, 3, 20523.

31 D. Ramirez, S. Y. Qi and M. J. Rood, Environ. Sci. Technol., 2005, 39, 5864-5871.

32 M. E. Ramos, P. R. Bonelli, A. L. Cukierman, M. M. L. R. Carrott and P. J. M. Carrott, J. Hazard. Mater., 2010, 177, 175-182.

33 P. Liu, C. Long, Q. F. Li, H. M. Qian, A. M. Li and Q. X. Zhang, J. Hazard. Mater., 2009, 166, 46-51.

34 X. J. Feng and L. Jiang, Adv. Mater., 2006, 18, 3063-3078.

35 N. Foley, K. Thomas, P. Forshaw, D. Stanton and P. Norman, Langmuir, 1997, 13, 2083-2089.

36 J. G. Bell, X. Zhao, Y. Uygur and K. M. Thomas, J. Phys. Chem. C, 2011, 115, 2776-2789.

37 D. D. Do and H. D. Do, Chem. Eng. J., 1997, 52, 297-310.

38 K. Kosuge, S. Kubo, N. Kikukawa and M. Takemori, Langmuir, 2007, 23, 3095-3102.

39 M. P. Cal, M. J. Rood and S. M. Larson, Gas Sep. Purif., 1996, 10, 117-121.

40 H.-C. Shin, J.-W. Park, K. Park and H.-C. Song, Environ. Pollut., 2002, 119, 227-236.

41 Q. Hu, B. J. Dou, H. Tian, J. J. Li, P. Li and Z. P. Hao, Microporous Mesoporous Mater., 2010, 129, 30-36. 